\title{
Global and Regional Changes in Cortical Development Assessed by MRI in Fetuses with Isolated Nonsevere Ventriculomegaly Correlate with Neonatal Neurobehavior
}

\author{
(D) N. Hahner, (D) O.M. Benkarim, (D) M. Aertsen, (D) M. Perez-Cruz, (D) G. Piella, D G. Sanroma, (D) N. Bargallo, (D). Deprest,
} (D) M.A. Gonzalez Ballester, (DE. Gratacos, and (DE. Eixarch

\begin{abstract}
BACKGROUND AND PURPOSE: Fetuses with isolated nonsevere ventriculomegaly (INSVM) are at risk of presenting neurodevelopmental delay. However, the currently used clinical parameters are insufficient to select cases with high risk and determine whether subtle changes in brain development are present and might be a risk factor. The aim of this study was to perform a comprehensive evaluation of cortical development in INSVM by magnetic resonance (MR) imaging and assess its association with neonatal neurobehavior.
\end{abstract}

MATERIALS AND METHODS: Thirty-two INSVM fetuses and 29 healthy controls between $26-28$ weeks of gestation were evaluated using MR imaging. We compared sulci and fissure depth, cortical maturation grading of specific areas and sulci and volumes of different brain regions obtained from 3D brain reconstruction of cases and controls. Neonatal outcome was assessed by using the Neonatal Behavioral Assessment Scale at a mean of $4 \pm 2$ weeks after birth.

RESULTS: Fetuses with INSVM showed less profound and underdeveloped sulcation, including the Sylvian fissure (mean depth: controls $16.8 \pm 1.9 \mathrm{~mm}$, versus INSVM $16.0 \pm 1.6 \mathrm{~mm} ; P=.01$ ), and reduced global cortical grading (mean score: controls $42.9 \pm 10.2 \mathrm{~mm}$, versus INSVM: $37.8 \pm 9.9 \mathrm{~mm} ; P=.01)$. Fetuses with isolated nonsevere ventriculomegaly showed a mean global increase of gray matter volume (controls, $276.8 \pm 46.0 \times 10 \mathrm{~mm}^{3}$, versus INSVM $277.5 \pm 49.3 \times 10 \mathrm{~mm}^{3}, P=.01$ ), but decreased mean cortical volume in the frontal lobe (left: controls, $53.2 \pm 8.8 \times 10 \mathrm{~mm}^{3}$, versus INSVM $\left.52.4 \pm 5.4 \times 10 \mathrm{~mm}^{3} ; P=<.01\right)$. Sulcal depth and brain volumes were significantly associated with the Neonatal Behavioral Assessment Scale severity $\left(P=.005\right.$, Nagelkerke $\left.R^{2}=0.732\right)$.

CONCLUSIONS: INSVM fetuses showed differences in cortical development, including regions far from the lateral ventricles, that are associated with neonatal neurobehavior. These results suggest the possible use of these parameters to identify cases at higher risk of altered neurodevelopment.

ABBREVIATIONS: INSVM = isolated nonsevere ventriculomegaly; NBAS = Neonatal Behavioral Assessment Scale; VM = ventriculomegaly

Cetal ventriculomegaly (VM) is defined as an enlargement of 1 or both lateral ventricles of $\geq 10 \mathrm{~mm}$ measured by sonography. ${ }^{1} \mathrm{VM}$ is the most common brain anomaly, occurring in

\section{Received May 13, 2019; accepted June 28}

From the Fetal i+D Fetal Medicine Research Center (N.H., M.P.-C., E.G., E.E.), BCNatalBarcelona Center for Maternal-Fetal and Neonatal Medicine (Hospital Clínic and Hospital Sant Joan de Déu), Institut Clínic de Ginecologia, Obstetricia i Neonatologia, Institut d'Investigacions Biomèdiques August Pi i Sunyer, Universitat de Barcelona, Barcelona, Spain; Centre for Biomedical Research on Rare Diseases (E.G., E.E.), Barcelona, Spain; Magnetic Resonance Image Core Facility (N.B.), Institut d'Investigacions Biomediques August Pi i Sunyer, Barcelona, Spain; Department of Radiology (N.B.), Centre de Diagnòstic per la Imatge, Hospital Clínic, Barcelona, Spain; BCN MedTech (O.M.B., G.P., G.S., M.A.G.B.), Universitat Pompeu Fabra, Barcelona, Spain; ICREA (M.A.G.B.), Barcelona, Spain; Departments of Radiology (M.A.) and Obstetrics (J.D.), UZ Leuven, Leuven, Belgium; and Institute for Women's Health (J.D.), University College London, London, UK.

The research leading to these results was partially funded by the Erasmus + Programme of the European Union (Framework Agreement No. 2013-0040), Instituto de Salud Carlos III (PI16/00861) integrados en el Plan Nacional de I+D+I y cofinanciados por el ISCIII-Subdirección General de Evaluación y el Fondo Europeo de Desarrollo Regional "Una manera de hacer Europa," CERCA Programme/Generalitat de Catalunya, "la Caixa" Foundation (LCF/PR/GN14/10270005), AGAUR 2017 SGR grant No. 1531, and The Cerebra Foundation for the Brain-Injured Child, Carmarthen, Wales. around $1 \%$ of pregnancies. ${ }^{2}$ In the absence of additional abnormalities such as aneuploidy, malformation or infections, which are found in approximately $50 \%$ of cases, VM is considered as

The publication reflects only the views of the authors, and the Commissions holder of the grants cannot be held responsible for any use that may be made of the information contained therein.

Paper previously presented orally at: Ultrasound Meets Magnetic Resonance, September 28-29, 2018, Paris, France; and The Fetal Medicine Foundation World Congress, June 24-28, 2018, Athens, Greece: Regional Differences of Cortical Grey Matter Development in Fetuses with Isolated Non-Severe Ventriculomegaly, Hahner N, Benkarim O, Piella G, Sanroma G, Bargallo N, Gonzalez Ballester MA, Grata$\cos$ E, Eixarch E; Poster presentation at: International Society of Ultrasound in Obstetrics and Gynecology Congress, September 16-17, 2017, Vienna, Austria: Differential Cortical Development in Fetuses with Isolated Non-Severe Ventriculomegaly Assessed by MRI, Hahner N, Puerto B, Perez-Cruz M, Gratacos E, Eixarch E. Please address correspondence to Eduard Gratacos, MD, BCNatal-Barcelona Center for Maternal-Fetal and Neonatal Medicine, Sabino de Arana 1, 08028 Barcelona, Spain; e-mail: gratacos@clinic.cat

- Indicates open access to non-subscribers at www.ajnr.org

三 Indicates article with supplemental on-line table.

Indicates article with supplemental on-line photos.

http://dx.doi.org/10.3174/ajnr.A6165 
isolated, and is, therefore, related to a good prognosis. ${ }^{3}$ However, even cases with isolated mild VM have a higher risk of abnormal neurodevelopment compared with the healthy population. ${ }^{3}$ Indeed, about $11 \%$ of VM cases present neurobehavioral problems, including motor ${ }^{4}$ and language dysfunction, ${ }^{5}$ cognitive delay, ${ }^{4,6}$ and psychiatric disorders. ${ }^{7-9}$ Ventricular width ${ }^{10}$ and progression of the $\mathrm{VM}^{11}$ are predictive factors of adverse outcomes. Nevertheless, these parameters cannot discriminate cases that will present neurodevelopmental impairment. Thus, early identification of individuals at high risk of abnormal neurodevelopment is crucial in order to ensure the implementation of early therapeutic interventions.

There is some evidence demonstrating altered brain development in VM in the prenatal and postnatal periods. Advanced magnetic resonance (MR) imaging modalities have been used to demonstrate global changes in brain structure such as altered white matter microstructure in neonates ${ }^{12}$ and increased gray matter volumes in fetuses, ${ }^{13}$ neonates, ${ }^{12}$ and children. ${ }^{14}$ Evaluation of cortical development by advanced sonography has demonstrated an inverse relation between calcarine and ventricular size, ${ }^{15}$ with depth being an independent marker of later progression. ${ }^{15}$ Recently, underdeveloped cortical maturation has been described in fetuses with isolated nonsevere VM (INSVM), including the mesial area and the Sylvian fissure, which are regions in which the effect of ventricular dilation is unlikely. ${ }^{16}$ Sonography is the most commonly used imaging tool in fetal medicine; however, it has important limitations in the evaluation of several brain regions, such as the fetal skull shadow which impedes the study of some cortical regions, especially the proximal hemisphere. On the contrary, MR imaging allows complete visualization of both hemispheres, and cortical maturation of the entire brain can be assessed.

The objective of this study was to perform a comprehensive assessment of cortical maturation by MR imaging, including sulci depth, cortical grading, and volume, and to evaluate the association with neonatal neurobehavior.

\section{MATERIALS AND METHODS \\ Subjects}

A prospective case-control study was performed in BCNatal (Hospital Clínic and Hospital Sant Joan de Déu) in Barcelona from 2014 to 2016. The Ethics Committee of our center approved the study protocol (HCB/2014/0484), and all patients and healthy volunteers gave written informed consent.

Nonsevere VM was defined as an atrial width between 10.0 and $14.9 \mathrm{~mm}$ at sonographic examination. Progression or regression was defined as a change of lateral ventricular width $\geq 2 \mathrm{~mm}$. Atrial width was assessed by measuring the distance between the inner border of both lateral walls at the height of the glomus and the parieto-occipital sulcus in the axial transventricular plane. ${ }^{17} \mathrm{Ges}-$ tational age was dated using the first trimester crown rump length measurement. All patients with an abnormal karyotype or microarray findings, infections, other structural abnormalities associated with higher risk of abnormal neurodevelopment or acquired ventriculomegaly were excluded. The control group was made up of pregnant volunteers with healthy and normally grow- ing fetuses of singleton pregnancies and were prospectively enrolled specifically for research proposes of this study.

\section{MR Imaging Acquisition}

MR imaging was performed at 26.0 to 28.6 gestational weeks. All images were obtained with a $1.5 \mathrm{~T}$ MR imaging (Magnetom Aera syngo MR D13; Siemens, Erlangen, Germany) using an 8-channel body coil without fetal sedation and following the American College of Radiology guidelines for pregnancy and lactation. ${ }^{8}$ The acquisition used for sulcal depth measurements and grading consisted in singleshot, fast spin-echo T2-weighted sequence $(\mathrm{TR}=1000 \mathrm{~ms}, \mathrm{TE}=137$ $\mathrm{ms}$, slice thickness $=3.5 \mathrm{~mm}$, no gap, FOV $=300 \times 225 \mathrm{~mm}$, voxel size $=1.17 \times 1.17 \times 3.5 \mathrm{~mm}$, matrix $=256 \times 192$, flip angle $=135^{\circ}$, acquisition time $=32 \mathrm{~s}$ ) acquired in the 3 orthogonal planes of the fetal head. For volumetric analyses, additional images of 4 axial, 2 coronal, and 2 sagittal single-shot fast spin-echo sequences (TR = $1500 \mathrm{~ms}$, TE $=82 \mathrm{~ms}$, slice thickness $=2.5 \mathrm{~mm}$, no gap, voxel size $=1.09 \times 1.09 \times 2.5 \mathrm{~mm}$, FOV $=280 \times 225 \mathrm{~mm}$, matrix $=$ $256 \times 256 \mathrm{~mm}$, flip angle $=121^{\circ}$, acquisition time $=47 \mathrm{~s}$ ) were obtained for later reconstruction. The sequences were repeated when the attending fetal medicine specialist (N.H.) observed insufficient image quality. All clinical images were reviewed by a specialized radiologist (N.B.) to exclude any additional abnormality of the central nervous system.

\section{MR Imaging Analysis}

Sulcal Depth. Sulcal measurement was performed off-line using Analyze 9.0 software (AnalyzeDirect, Overland Park, Kansas) for T2-weighted sequences with a $3.5-\mathrm{mm}$ slice thickness. First, laterality was assessed by the fetus's position in utero, and both hemispheres were evaluated. Selection of anatomic planes for 2D measurements was assessed according to sonography guidelines. ${ }^{17}$ Sulci were measured using previously described methodology. ${ }^{18,19}$ The measurements were normalized by biparietal diameter (BPD) and multiplied by 100. A midline was drawn for each plane from the frontal towards the occipital skull projecting the interhemispheric fissure in axial views, and from cranial to caudal to highlight the fissure in coronal views. Sulcal and fissure depth was obtained as previously reported ${ }^{18}$ by drawing a perpendicular line from the midline toward the border of the specific sulcus as shown in Fig $1 A-D$.

Cortical Grading. Cortical areas and sulci were graded according to previously described methodology. ${ }^{20}$ The grading scheme scores all main cortical areas (frontal, parietal, temporal, occipital, and mesial cortical area), sulci (parieto-occipital, superior temporal, central, calcarine, and cingulate sulci), and the Sylvian fissure in a scale from 0 (no maturation) to 5 (maximum degree of maturation) (Fig $1 E-L$ ). Subsequently, the scores of all the areas and sulci on each side were considered to calculate the hemispheric and total brain grading scores.

Cortical Volumes. To perform volumetric analyses of the cortex, the fetal brain was first located and extracted from the previously mentioned image stacks using an automatic approach proposed by Keraudren et al. ${ }^{21}$ Then, high-resolution $3 \mathrm{D}$ volume reconstruction with a super-resolution of $0.75 \times 0.75 \times 0.75 \mathrm{~mm}$ was obtained according to the method of Kuklisova-Murgasova et al. ${ }^{22}$ 


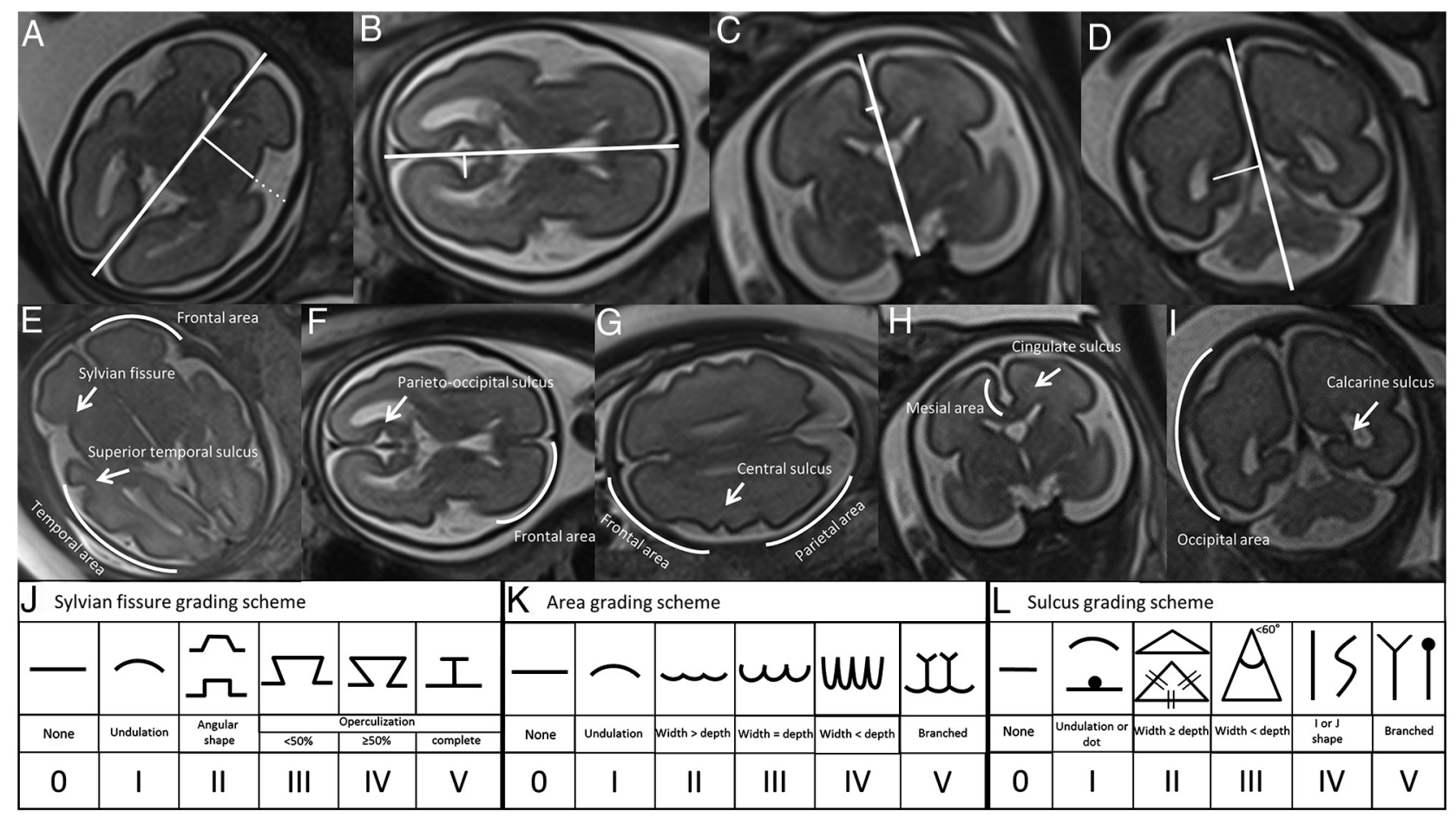

FIG 1. Assessment of sulcal depth and cortical grading. Sulcal depth: The bold white interhemispheric line is used as reference for all the measurements and the thin white line shows the measurement of the sulcus of interest, including the insula and Sylvian fissure (dotted line) in the axial transthalamic plane $(A)$, parieto-occipital sulcus in the axial transventricular plane $(B)$, cingulate sulcus in the coronal transthalamic view $(C)$, and the calcarine sulcus in the coronal transcerebellar plane $(D)$. Cortical grading: Curved lines indicate the areas, and arrows point out the sulci of interest. In the transthalamic plane frontal and temporal area $(E)$, the Sylvian fissure and superior temporal sulcus were evaluated. In the transventricular plane $(F)$, the parieto-occipital sulcus and frontal area were assessed, and in the plane superior to it (G), the central sulcus and frontal and parietal areas were assessed. The mesial area and cingulate sulcus were evaluated in the coronal transthalamic plane $(H)$, and the calcarine sulcus was assessed in the coronal transcerebellar plane (l). Scheme: grading scale for Sylvian fissure (l), cortical areas $(K)$ and sulci $(L)$ by Pistorius et al. ${ }^{20}$

Brain tissue was automatically segmented from these reconstructed volumes into white matter, cortex, cerebrospinal fluid (CSF), ventricles, cerebellum, and brainstem. This was done using an ensemble method reported by Sanroma et al, ${ }^{23}$ which combines 2 base approaches. All segmentations were further revised and manually corrected if necessary by 2 experts (N.H. and E.E.).

Taking into account that volumetric analyses of the cortex were the focus of the study, the cortex was parcellated into 16 regions along the cortical surface. ${ }^{24}$ Figure 2 and On-line Figure 1 show the steps from fetal head extraction to parcellation.

\section{Neonatal Neurobehavior}

Neonatal neurobehavior was assessed at a mean of $4 \pm 2$ weeks after birth using the Neonatal Behavioral Assessment Scale (NBAS) developed by Brazelton and Nugent. ${ }^{25}$ This test evaluates the capacity of neonates to interact with the environment and provides information about brain maturation. ${ }^{26}$ The 2 observers who performed the test were accredited by the Brazelton Institute (Harvard Medical School, Boston, Massachusetts) and blinded to the identification of cases and controls. The evaluation was made in a small, quiet, semidark, warm room with at least 1 parent present. The scale consists of 35 items, rated from 1 to 9 points, and, are summarized into 6 clusters (habituation, motor system, social-interactive, range of states, regulation of states, and autonomic stability). Cluster scores were transformed into $z$ scores according to a standard population ${ }^{27}$ and were defined as abnor- mal if the $z$ score was below -1 . The NBAS severity score was defined as the number of abnormal NBAS clusters in each subject.

\section{Statistical Analyses}

Data analyses were performed using SPSS, Version 22.0 for Windows (IBM, Armonk, New York). Normality was tested using the Shapiro-Wilk test. The Student's $t$ test for independent samples and the Pearson $\chi^{2}$ test were used to compare quantitative and qualitative data between those with INSVM and controls. For quantitative variables, a general linear model was carried out, and for categoric variables, ordinal regression was used. To correct for head size, we adjusted sulcal depth by biparietal diameter and volumetric analyses by supratentorial volume. Prenatal data were additionally adjusted by sex and gestational age at MR imaging. Neonatal neurobehavior data were adjusted for sex, breastfeeding, socioeconomic status, gestational age at neonatal examination, and days of neonatal adaption. The association between prenatal parameters and the NBAS severity score was assessed by ordinal regression.

To assess the reproducibility of cortical grading and sulcal depth using MR imaging, a second observer (M.A.) blinded to the measurements of the first observer applied the same methodolgy in 20 fetuses using MeVisLab software (MeVis Medical Solutions, Bremen, Germany). Complete MR imaging sequences of each fetus were given to the second observer to select the appropriate images to measure. The 


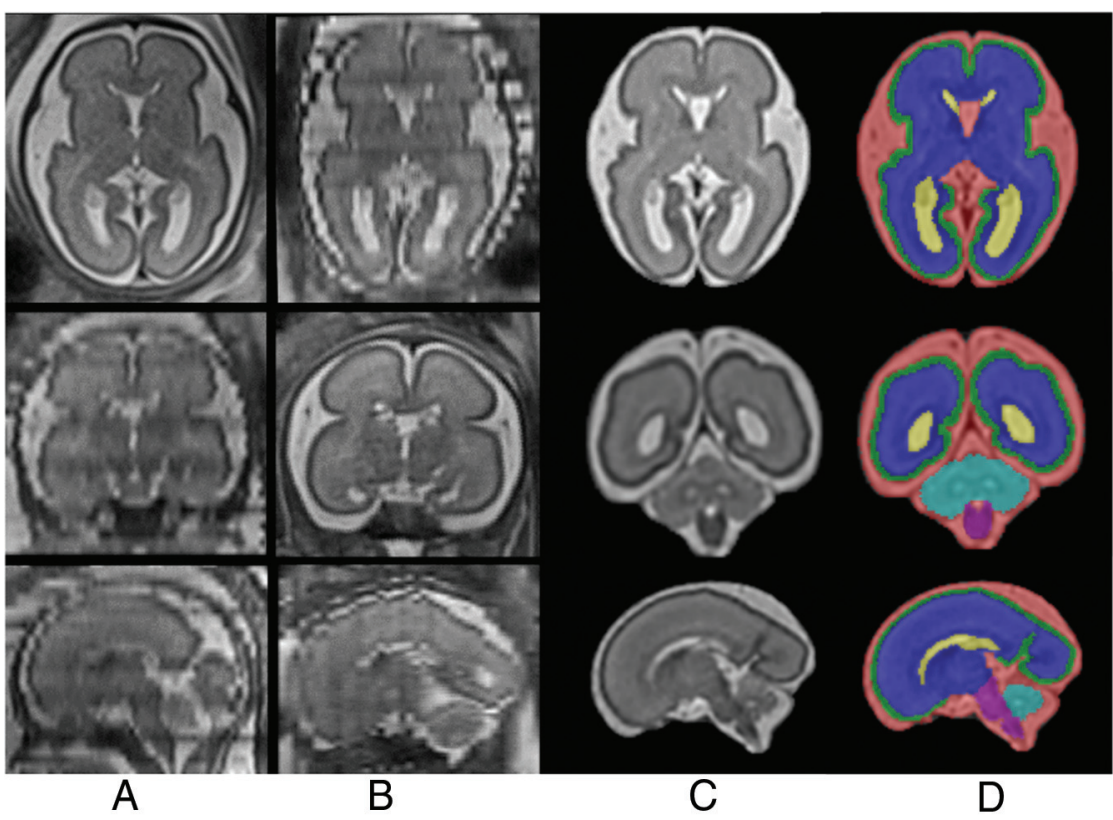

FIG 2. Steps of fetal brain MR imaging reconstruction and tissue segmentation. Columns $A$ and $B$, The raw stacks, including the image obtained as a reference (Column $A$, axial, and Column $B$, coronal) and the orthogonal views (note the low quality of these images). Column $C$, The 3 orthogonal planes of the final reconstruction. Column $D$, The segmentation into cortical gray matter (green), white matter (blue), lateral ventricles (yellow), CSF outside the ventricles (red), cerebellum (turquoise), and brain stem (purple).

interclass correlation coefficient was calculated using a 2-way random effect model with absolute agreement.

\section{RESULTS}

\section{Subjects}

Thirty-three cases of nonsevere VM diagnosed before 28 weeks of gestation were included in our cohort. In 1 case, microarray identified a 16p11.2 duplication is associated with a higher risk of neurodevelopmental disorders and this case was excluded from the cohort. All other fetuses with VM had normal karyotype $(n=$ $17)$ or micro-array $(n=13)$ findings determined by amniocentesis. In 2 cases, parents refused to perform amniocentesis, and a normal microarray findings were obtained in a genetic study using saliva sampling in the postnatal period. Infection was excluded by negative polymerase chain reaction findings in amniotic liquid in 30 cases and in the remaining ones by maternal serologic screening. The control group included 32 healthy fetuses with normal growth. Of these, 2 mothers finally could not attend the MR imaging appointment, and 1 acquisition had to be excluded due to bad image quality, resulting in a final sample size of 32 fetuses with INSVM and 29 controls. The demographic characteristics of the study groups are presented in Table 1.

\section{MR Imaging Data}

Sulcal depth measurement and cortical grading evaluation was performed in $98.4 \%$ of examinations; only 1 control subject had insufficient image quality and was not considered for final analyses. $3 \mathrm{D}$ reconstructions and volumetric analyses were performed in $78.7 \%$ of the fetuses.

Sulcal Depth. Table 2 shows the comparison of the measurements of the INSVM fetuses and the controls. As expected, fetuses with INSVM showed significantly less profound parieto-occipital and calcarine sulci in both hemispheres compared with controls. In addition to these differences in sulci near the ventricles, those with INSVM showed increased insula depths in both hemispheres and a less profound Sylvian fissures in the right hemisphere. No significant differences were found in the cingulate sulcus at this gestational age.

When only bilateral cases were considered, differences were maintained and changes in the Sylvian fissure mean measurement were also observed in the left hemisphere (left: controls, $16.7 \pm$ $1.6 \mathrm{~mm}$, versus INSVM, $15.3 \pm 2.3 \mathrm{~mm}$; $P=.02$; right: controls, $16.8 \pm 1.9 \mathrm{~mm}$, versus INSVM, $15.5 \pm 2.0 \mathrm{~mm} ; P=.06$ ).

Cortical Grading. In general, fetuses with INSVM had lower cortical grading scores compared with the control group. Parieto-occipital and calcarine sulci showed delayed grading in both hemispheres (left parieto-occipital sulcus grading, $\geq 3$ : controls, $37.9 \%$, versus INSVM, $15.2 \%, P=$ .03 ; right: controls, $58.6 \%$, versus INSVM, 30.6\%; $P=.02$ ) (left calcarine sulcus grading, $\geq 4$ : controls, $55.1 \%$, versus INSVM, $24.2 \%$; $P=<.01$; right: controls, $65.5 \%$, versus INSVM, $27.3 \% ; P=<.01$ ). Furthermore, significantly altered maturation in the left superior temporal sulcus showed (grading, $\geq 2$ : controls, $55.2 \%$, versus INSVM, $27.7 \% ; P=.04$ ). In the subgroups of fetuses with bilateral INSVM, we identified more marked differences compared with unilateral cases, with significant delay in the right parietal (grading, $\geq 2$ : controls, $65.5 \%$, versus INSVM, $18.2 \% ; P=.03$ ) and occipital areas (grading, $\geq 2$ : controls, $62.0 \%$, versus INSVM, $9.1 \% ; P=.2$ ). Finally, considering the sulcation pattern of the whole hemisphere, a significant difference was observed in fetuses with INSVM on both the right and left compared to the control group (Table 2).

Interobserver Agreement. Overall, cortical development assessment showed a good interobserver reproducibility with an interclass correlation coefficient of 0.962 (95\% CI, 0.950-0.971) for depth measurements and 0.759 (95\% CI, 0.717-0.796) for cortical grading.

Cortical Volumes. Volumetric analyses of 3D reconstructed MR imaging showed, generally greater brain tissue volumes (supratentorial, cortex, white matter, lateral ventricle), with significant differences in cortical and ventricular volumes in the INSVM group (Fig 3). Indeed, as shown in On-line Figure 2, ventricular volume was positively correlated with brain tissue volumes. Regional evaluation identified a significantly lower mean cortical volume in both frontal lobes in the INSVM compared with the control group (left: controls, $53.2 \pm 8.8 \times 10 \mathrm{~mm}^{3}$, versus INSVM, $52.4 \pm 5.4 \times 10 \mathrm{~mm}^{3} ; P=<.01$; right: controls, $52.8 \pm$ $9.5 \times 10 \mathrm{~mm}^{3}$, versus INSVM, $51.0 \pm 9.9 \times 10 \mathrm{~mm}^{3} ; P=<.01$ ) and greater volumes of the posterior part of the cingulate gyri in 
Table 1: Clinical characteristics of the study groups ${ }^{\mathrm{a}}$

\begin{tabular}{|c|c|c|c|}
\hline & $\begin{array}{l}\text { Controls } \\
(n=29)\end{array}$ & $\begin{array}{l}\text { INSVM } \\
(n=32)\end{array}$ & $P$ \\
\hline Maternal age (yr) & $33.7 \pm 4.2$ & $32.4 \pm 5.5$ & .32 \\
\hline Birth weight (g) & $3414 \pm 517$ & $3406 \pm 553$ & .96 \\
\hline Gestational age at birth (wk) & $39.8 \pm 0.9$ & $39.8 \pm 1.2$ & .81 \\
\hline Gestational age at MRI (wk) & $27.7 \pm 0.9$ & $27.7 \pm 0.9$ & .91 \\
\hline Lateral ventricular width ${ }^{\mathrm{a}}$ & $4.6 \pm 1.4$ & $10.6 \pm 1.1$ & $<.01^{\mathrm{b}}$ \\
\hline Fetus sex & & & $.02^{\mathrm{c}}$ \\
\hline Male & $58.6 \%(17)$ & $87.5 \%(28)$ & \\
\hline Female & $41.4 \%(12)$ & $12.5 \%(4)$ & \\
\hline \multicolumn{4}{|l|}{ Laterality of ventriculomegaly } \\
\hline Bilateral & - & $34.4 \%(11)$ & \\
\hline Unilateral left & - & $34.4 \%(11)$ & \\
\hline Unilateral right & - & $31.2 \%(10)$ & \\
\hline \multicolumn{4}{|c|}{ Evolution of lateral ventricular width ${ }^{c}$} \\
\hline Regressive & - & $25 \%(8)$ & \\
\hline Stable & - & $75 \%(24)$ & \\
\hline Progressive & - & $0 \%(0)$ & \\
\hline \multicolumn{4}{|c|}{ Classification according to atrial width } \\
\hline Mild $(10.0-11.9 \mathrm{~mm})$ & - & $84.3 \%(27)$ & \\
\hline Moderate $(12.0-13.9 \mathrm{~mm})$ & - & $16.7 \%(5)$ & \\
\hline
\end{tabular}

Note:- - indicates that these characteristics do not apply for the control group.

${ }^{a}$ Comparison of clinical characteristics between the control and case cohort. Results are expressed as means or percentage and number of subjects as appropriate.

${ }^{b}$ Measurement of the more dilated lateral ventricle by ultrasound at diagnosis.

'Evolution of lateral ventricular width until term of pregnancy.

${ }^{\mathrm{d}}$ Significant ( $\left.\leq .05\right)$

Table 2: Sulcal depth and grading scores of the study groups ${ }^{\mathrm{a}}$

\begin{tabular}{lccc}
\hline \multicolumn{1}{c}{ Variable } & $\begin{array}{c}\text { Controls } \\
(\boldsymbol{n}=\mathbf{2 9})\end{array}$ & $\begin{array}{c}\text { INSVM } \\
(\boldsymbol{n}=\mathbf{3 2})\end{array}$ & $\boldsymbol{P}$ \\
\hline $\begin{array}{l}\text { Left hemisphere depths } \\
\text { Insula }\end{array}$ & $29.4 \pm 1.5$ & $29.7 \pm 1.8$ & $.03^{\mathrm{b}}$ \\
Sylvian fissure & $16.7 \pm 1.6$ & $17.2 \pm 2.4$ & .15 \\
Parieto-occipital sulcus & $10.2 \pm 2.3$ & $8.1 \pm 2.6$ & $<.01^{\mathrm{b}}$ \\
Cingulate sulcus & $3.8 \pm 1.3$ & $4.3 \pm 2.5$ & .29 \\
Calcarine sulcus & $12.5 \pm 2.7$ & $10.9 \pm 2.1$ & $<.01^{\mathrm{b}}$ \\
Right hemisphere depths & & & $.04^{\mathrm{b}}$ \\
Insula & $29.6 \pm 1.4$ & $29.7 \pm 1.7$ & $.01^{\mathrm{b}}$ \\
Sylvian fissure & $16.8 \pm 1.9$ & $16.0 \pm 1.6$ & $<.01^{\mathrm{b}}$ \\
Parieto-occipital sulcus & $11.1 \pm 2.4$ & $9.0 \pm 2.8$ & .33 \\
Cingulate sulcus & $3.6 \pm 1.1$ & $4.0 \pm 1.5$ & $<.02^{\mathrm{b}}$ \\
Calcarine sulcus & $13.5 \pm 2.2$ & $11.8 \pm 4.8$ & $.01^{\mathrm{b}}$ \\
Sum of grading scores & & & $.03^{\mathrm{b}}$ \\
Left hemisphere & $21.1 \pm 5.3$ & $18.2 \pm 5.1$ & $.01^{\mathrm{b}}$ \\
Right hemisphere & $21.8 \pm 5.1$ & $19.6 \pm 5.3$ & $37.8 \pm 9.9$ \\
Total cortex & $42.9 \pm 10.2$ & & \\
\hline
\end{tabular}

${ }^{a}$ Comparison of sulcal depth measurements between the control and case cohort. Depth measurements (in millimeters) were normalized by BPD and multiplied by 100 . Results are expressed as means. $P$ values were adjusted by gestational age at examination and sex.

${ }^{\mathrm{b}}$ Values $\leq .05$

both hemispheres (left: controls, $1.5 \pm 0.2 \times 10 \mathrm{~mm}^{3}$, versus INSVM, $1.7 \pm 0.4 \times 10 \mathrm{~mm}^{3} ; P=.01$; right, $1.6 \pm 0.2 \times 10 \mathrm{~mm}^{3}$, versus INSVM, $\left.1.9 \pm 0.4 \times 10 \mathrm{~mm}^{3} ; P=.03\right)$. In addition, fetuses with INSVM had a decreased mean volume in the anterior part of the gyri parahippocampalis and ambiens of the left hemisphere (controls, $3.4 \pm 0.5 \times 10 \mathrm{~mm}^{3}$ versus $3.3 \pm 0.5 \times 10 \mathrm{~mm}^{3} ; P=$ $.04)$. On-line Table 1 shows all the data collected in the regional cortical volume analysis.

\section{Neonatal Neurobehavior}

The NBAS could be performed in only 24 fetuses with INSVM and 24 controls ( $75 \%$ and $82.8 \%$, respectively) because parents could not be contacted or refused to participate. Routine neonatal clin- ical examination findings were normal in all controls. The mean age at NBAS did not significantly differ between the two groups (controls, $44.5 \pm 2.1$ postmenstrual weeks, versus INSVM, $44.6 \pm$ 2.9 postmenstrual weeks; $P=.94$ ).

The habituation cluster was only completed in $37.5 \%$ of cases $(n=9)$ and was not considered for the analysis. After we adjusted for confounding variables, no significant differences were found between groups. A tendency of weaker performance in the case group was observed in the motor cluster (mean $z$ scores: controls, $0.74 \pm 0.71$, versus INSVM $0.32 \pm 0.86 ; P=.644$ ) and the range of state clusters (mean $z$ scores: controls, $-0.19 \pm 1.54$, versus INSVM $-0.32 \pm 1.41 ; P=.924)$, whereas slightly higher values were observed in the social-interactive cluster (mean $z$ scores: controls, $0.71 \pm 0.67$, versus INSVM $0.78 \pm 0.65 ; P=.091$ ).

To explore the association between prenatal parameters and neonatal neurobehavior, we performed ordinal regression of the NBAS severity score (dependent variable) with main clinical data (study group, atrial diameter, bilateral dilation), yielding a nonsignificant model $\left(P=.768, \chi^{2}=1.826\right.$, Nagelkerke $\left.\mathrm{R}^{2}=0.032, d f=4\right)$. The addition of sulcal depth (insula, Sylvian fissure, and parieto-occipital, calcarine, and cingulate sulci) resulted in a nonsignificant model $\left(P=.115, \chi^{2}=20.508\right.$, Nagelkerke $\left.\mathrm{R}^{2}=0.308, d f=14\right)$ but increased the explanation of variability between controls and cases from $2.6 \%$ to $38.9 \%$. Finally, the addition of volumetric cortical data (total supratentorial volume (STV), total GM, left and right frontal lobes, left and right posterior part of the cingulate gyrus, left anterior part of the gyri parahippocampalis and ambiens), result in a statistically significant model $\left(P=.005, \chi^{2}=\right.$ 41.590, Nagelkerke $\mathrm{R}^{2}=0.732, d f=21$ ), showing a significant association of prenatal cortical parameters with the severity of abnormal neurobehavioral outcomes, providing significant additional information to the main clinical characteristics.

\section{DISCUSSION}

To the best of our knowledge, this is the first study to describe global and regional differences in cortical development assessed by MR imaging in fetuses with true INSVM and their correlation with neonatal neurobehavior. These differences were not only limited to regions adjacent to the lateral ventricles but also in- 

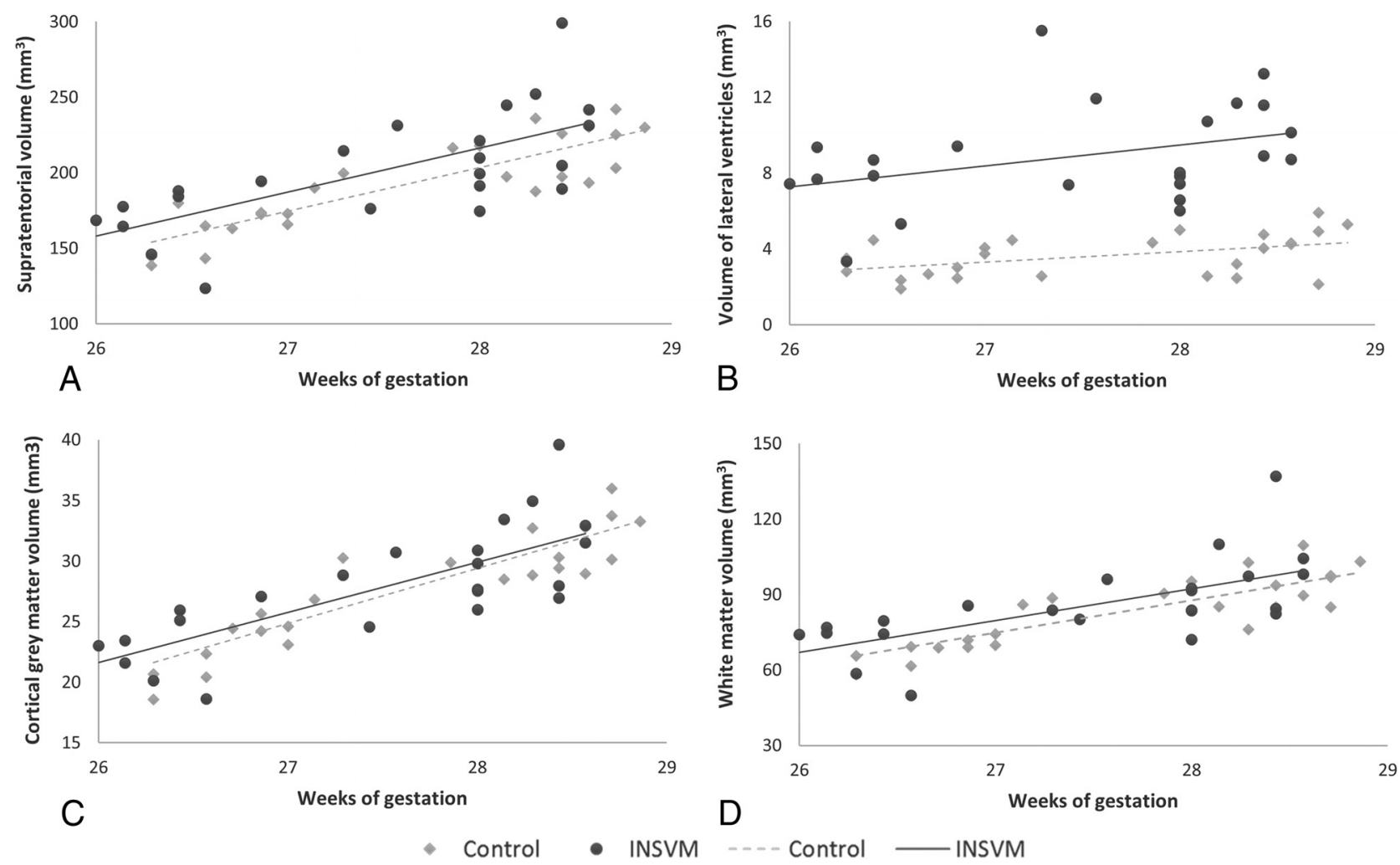

FIG 3. Brain tissue volumes in study groups. Cerebral tissue volumes including both hemispheres in INSVM (circles) and control (rhombuses) cohorts. A, Supratentorial volumes. B, Volumes of the lateral ventricles. $C$, Cortical gray matter volumes. $D$, White matter volumes. Volume measures are in cubic millimeters.

cluded the Sylvian fissure, cingulate gyrus, and the frontal, temporal and occipital regions.

Our results are in line with previous data showing significant differences in development of the parieto-occipital and calcarine sulci ${ }^{15}$ and the Sylvian fissure and insula using sonography. ${ }^{16}$ It should be noted that the Sylvian fissure and insula are involved in speech and language processing and influence pathways of cognition and emotion, ${ }^{28,29} 2$ functions that have been described as being altered in VM. ${ }^{4,6}$ Nevertheless, this study adds valuable information providing a comprehensive evaluation of global and regional cortical development using different features of prenatal MR imaging, including sulcal depth, grading, and cortical volumes. Global cortical grading was significantly lower in INSVM in both hemispheres, reflecting a general delayed brain development in these fetuses. Additionally, changes in several regions were found, which might explain neurodevelopmental problems presented by a subgroup of patients with INSVM. Among these regions, fetuses with INSVM presented significantly underdeveloped temporal sulcation. Indeed, this region is a key structure in language function and takes part in processing social cognition, which plays a role in subtypes of autism. ${ }^{30}$ Most interesting, bilateral cases presented widespread changes with delayed development in parietal, temporal, and occipital areas, the Sylvian fissure, and superior temporal sulci, which may reflect a more severe condition.

Several studies in the pre- and postnatal periods have demonstrated greater cortical volumes in mild fetal $\mathrm{VM}^{12-14}$ and our data confirm these findings, with a significant increase in cortical gray matter volume. It has been suggested that greater cortical volume is related to increased surface area along the enlarged ventricular wall, which may result in a larger number of progenitor neurons, ${ }^{14}$ or be due to the lack of regular apoptosis in the developing brain. ${ }^{13}$ Regional analysis showed that this enlargement was present in most regions, reaching significance in the posterior part of the cingulate gyrus and the anterior part of the gyri parahippocampalis and ambiens. On the other hand, decreased cortical volumes were found in the frontal lobe of both hemispheres. The frontal lobe is composed of several cytoarchitectural subregions, among them, the superior and medial frontal gyri, which are involved in specific functions such as cognition-related processing and are part of the default network, which is essential for normal cognition and self-referential processing. ${ }^{31}$ Most interesting, these frontal regions with reduced cortical volume are highly connected to the cingulate cortex, showing greater volumes. We hypothesize that these volumetric changes might reflect cytoarchitectonic changes, which could imply changes in interaction and functionality.

The prognosis for INSVM is considered good in around $90 \%$ of the cases. ${ }^{3}$ However, within this pooled risk, there is a wide range of abnormal outcomes from $0 \%{ }^{32}$ to $36 \% .{ }^{33}$ We failed to demonstrate statistically significant differences in neonatal neurobehavior, but INSVM cases showed weaker performance in the motor and range of state clusters. Nonetheless, we found a significant association between cortical development parameters and NBAS results, explaining more than $70 \%$ of the variation in neurodevelopment in INSVM. Previous data have demonstrated the correlation between cortical devel- 
opment and later a neurobehavior in fetuses with congenital heart disease ${ }^{34}$ and fetal growth restriction. ${ }^{35}$

We acknowledge that this study has some limitations and strengths that should be discussed. First, although MR imaging is not affected by a fetal skull shadow, it has less spatial resolution than high-frequency transvaginal sonography; making tissue borders slightly blurred and less clear compared with sonography, this may have interfered in those sulci in the initial stage of development, such as the cingulate sulci. This finding could also explain the lower interclass correlation coefficient we obtained for the mesial area and cingulate sulcus and the absence of differences in this region. Second, the use of automatic quantification for cortical volumes could be influenced by segmentation of cortical tissue. To overcome this, we reviewed all images and corrected them manually if necessary. And finally, the lack of statistical differences in neurodevelopmental tests between these 2 study groups could be explained by the sample size and the fact that INSVM is a nonhomogeneous population in which only part of the group has abnormal neurodevelopment; therefore, differences between controls and fetuses with INSVM could be weaker. Although in the neonatal stage many neurodevelopmental functions can still not be assessed, several studies have highlighted evidence that neonatal behavioral skills are linked to later neurocognitive development. ${ }^{26}$

Considering strengths, this is the first study performing a comprehensive assessment of cortical development in a wellselected cohort of INSVM, including 3 different and complementary techniques to quantify cortical development by MR imaging. The methodologies applied are able to identify subtle changes that might not be detected in a standard clinical evaluation and, additionally, provide objective and quantitative data that can be used to develop biomarkers. Moreover, the use of MR imaging allows adequate visualization of both hemispheres, which is commonly limited when performing sonography. Finally, all cases included in this study were strictly selected, ruling out the genetic anomalies and/or fetal infection, resulting in a unique cohort of true INSVM, preventing the inclusion of conditions that could potentially involve brain alterations and bias our results.

From a clinical point of view, the results of this study provide new data suggesting that detailed evaluation of cortical development in INSVM could add predictive value to identify the cases at higher risk of presenting altered neurodevelopment. This finding is of great interest because in our cohort, clinical prognostic markers, including ventricular width and volume and their evolution, were not associated with neonatal neurobehavior. In addition, evaluation of sulcal depth and grading by MR imaging is feasible in clinical settings, and although our results are far from achieving individual prediction, they do provide seminal evidence to continue investigating the use of cortical parameters as imaging biomarkers.

\section{CONCLUSIONS}

This is a pioneering study that describes differences of cortical development assessed by MR imaging in fetuses with INSVM that are correlated with neonatal neurobehavior. These results provide the opportunity to explore the utility of these parameters to iden- tify cases of INSVM at higher risk of presenting altered neurodevelopment and who could therefore, benefit from early intervention. Further studies are needed to explore whether cortical development is also correlated with neurodevelopment later in life.

\section{ACKNOWLEDGMENTS}

We thank Magnetic Resonance Imaging Core Facility of the Institut d'Investigacions Biomediques August Pi i Sunyer for their technical help.

Disclosures: Nadine Hahner-RELATED: Grant: Agéncia de Gestió d'Ajusts Universitaris i de Recerca, SGR grant No. 1531; CERCA Programme Generalitat de Catalunya; The Cerebra Foundation for the Brain-Injured Child, Carmarthen Wales; European Commission Education, Audiovisual and Culture Executive Agency, 2013-0040; Ministerio de Economia y Competividad-Instituto de Salud Carlos III, PI16/00861; "La Caixa" Foundation, LCF/PR/GN14/10270005*; Support for Travel to Meetings for the Study or Other Purposes: European Commission Education, Audiovisual and Culture Executive Agency, 2013-0040.* Jan Deprest—RELATED: Grant: European Commission Education, Audiovisual and Culture Executive Agency, 2013-0040; UNRELATED: Board Membership: Editorial Board of Gynaecological Surgery. * Eduard GratacosRELATED: Grant: Agéncia de Gestió d'Ajusts Universitaris i de Recerca, SGR grant No. 1531; CERCA Programme Generalitat de Catalunya; The Cerebra Foundation for the Brain-Injured Child, Carmarthen, Wales; European Commission Education, Audiovisual and Culture Executive Agency, 2013-0040; Ministerio de Economia y Competividad-Instituto de Salud Carlos III, PI16/00861; "La Caixa" Foundation, LCF/PR/GN14/ 10270005. * * Money paid to institution.

\section{REFERENCES}

1. Cardoza JD, Goldstein RB, Filly RA. Exclusion of fetal ventriculomegaly with a single measurement: the width of the lateral ventricular atrium. Radiology 1988;169:711-14 CrossRef Medline

2. Salomon LJ, Bernard JP, Ville Y. Reference ranges for fetal ventricular width: a non-normal approach. Ultrasound Obstet Gynecol 2007;30:61-66 CrossRef Medline

3. Melchiorre $\mathrm{K}$, Bhide $\mathrm{A}$, Gika $\mathrm{AD}$, et al. Counseling in isolated mild fetal ventriculomegaly. Ultrasound Obstet Gynecol 2009;34:212-24 CrossRef Medline

4. Leitner Y, Stolar O, Rotstein M, et al. The neurocognitive outcome of mild isolated fetal ventriculomegaly verified by prenatal magnetic resonance imaging. Am J Obstet Gynecol 2009;201:215.e1-6 CrossRef Medline

5. Ouahba J, Luton D, Vuillard E, et al. Prenatal isolated mild ventriculomegaly: outcome in 167 cases. BJOG 2006;113:1072-79 CrossRef Medline

6. Sadan S, Malinger G, Schweiger A, et al. Neuropsychological outcome of children with asymmetric ventricles or unilateral mild ventriculomegaly identified in utero. BJOG 2007;114:596-602 CrossRef Medline

7. Gilmore JH, van Tol J, Kliewer MA, et al. Mild ventriculomegaly detected in utero with ultrasound: clinical associations and implications for schizophrenia. Schizophr Res 1998;33:133-40 CrossRef Medline

8. Tremblay E, Therasse E, Thomassin-Naggara I, et al. Quality initiatives: guidelines for use of medical imaging during pregnancy and lactation. RadioGraphics 2012;32:897-911 CrossRef Medline

9. Lyoo IK, Noam GG, Lee CK, et al. The corpus callosum and lateral ventricles in children with attention-deficit hyperactivity disorder: a brain magnetic resonance imaging study. Biol Psychiatry 1996;40: 1060-63 CrossRef Medline

10. Vergani P, Locatelli A, Strobelt N, et al. Clinical outcome of mild fetal ventriculomegaly. Am J Obstet Gynecol 1998;178:218-22 CrossRef Medline

11. Gaglioti P, Danelon D, Bontempo S, et al. Fetal cerebral ventriculomegaly: outcome in $\mathbf{1 7 6}$ cases. Ultrasound Obstet Gynecol 2005;25:372-77 CrossRef Medline

12. Lockwood Estrin G, Kyriakopoulou V, Makropoulos A, et al. Altered 
white matter and cortical structure in neonates with antenatally diagnosed isolated ventriculomegaly. Neuroimage Clin 2016;11: 139-48 CrossRef Medline

13. Kyriakopoulou V, Vatansever D, Elkommos S, et al. Cortical overgrowth in fetuses with isolated ventriculomegaly. Cereb Cortex 2014;24:2141-50 CrossRef Medline

14. Lyall AE, Woolson S, Wolfe HM, et al. Prenatal isolated mild ventriculomegaly is associated with persistent ventricle enlargement at ages 1 and 2. Early Hum Dev 2012;88:691-98 CrossRef Medline

15. Miguelote RF, Vides B, Santos RF, et al. Cortical maturation in fetuses referred for 'isolated' mild ventriculomegaly: a longitudinal ultrasound assessment. Prenat Diagn 2012;32:1273-81 CrossRef Medline

16. Hahner N, Puerto B, Perez-Cruz M, et al. Altered cortical development in fetuses with isolated nonsevere ventriculomegaly assessed by neurosonography. Prenat Diagn 2018;38:365-75 CrossRef Medline

17. International Society of Ultrasound in Obstetrics \& Gynecology Education Committee. Sonographic examination of the fetal central nervous system: guidelines for performing the 'basic examination' and the 'fetal neurosonogram.' Ultrasound Obstet Gynecol 2007;29: 109-16 CrossRef Medline

18. Egaña-Ugrinovic G, Sanz-Cortes M, Figueras F, et al. Differences in cortical development assessed by fetal MRI in late-onset intrauterine growth restriction. Am J Obstet Gynecol 2013;209:126.e1-8 CrossRef Medline

19. Alonso I, Borenstein M, Grant G, et al. Depth of brain fissures in normal fetuses by prenatal ultrasound between 19 and 30 weeks of gestation. Ultrasound Obstet Gynecol 2010;36:693-99 CrossRef Medline

20. Pistorius LR, Stoutenbeek P, Groenendaal F, et al. Grade and symmetry of normal fetal cortical development: a longitudinal two- and three-dimensional ultrasound study. Ultrasound Obstet Gynecol 2010;36:700-08 CrossRef Medline

21. Keraudren K, Kuklisova-Murgasova M, Kyriakopoulou V, et al. Automated fetal brain segmentation from 2D MRI slices for motion correction. Neuroimage 2014;101:633-43 CrossRef Medline

22. Kuklisova-Murgasova M, Quaghebeur G, Rutherford MA, et al. Reconstruction of fetal brain MRI with intensity matching and complete outlier removal. Med Image Anal 2012;16:1550-64 CrossRef Medline

23. Sanroma G, Benkarim OM, Piella G, et al. Building an ensemble of complementary segmentation methods by exploiting probabilistic estimates. In: Proceedings of the International Workshop on Machine Learning in Medical Imaging, Athens, Greece. October 17, 2016; Vol 10019 LNCS.;27-35

24. Benkarim OM, Hahner N, Piella G, et al. Cortical folding alterations in fetuses with isolated non-severe ventriculomegaly. Neuroimage Clin 2018;18:103-14 CrossRef Medline

25. Brazelton TB, Nugent JK. Neonatal Behavioral Assessment Scale. London: McKeith Press; 1995

26. Canals J, Hernández-Martínez C, Esparó G, et al. Neonatal Behavioral Assessment Scale as a predictor of cognitive development and IQ in full-term infants: a 6-year longitudinal study. Acta Paediatr 2011;100:1331-37 CrossRef Medline

27. Boatella-Costa E, Costas-Moragas C, Botet-Mussons F, et al. Behavioral gender differences in the neonatal period according to the Brazelton scale. Early Hum Dev 2007;83:91-97 CrossRef Medline

28. Gasquoine PG. Contributions of the insula to cognition and emotion. Neuropsychol Rev 2014;24:77-87 CrossRef Medline

29. Peschke C, Ziegler W, Eisenberger J, et al. Phonological manipulation between speech perception and production activates a parietofrontal circuit. Neuroimage 2012;59:788-99 CrossRef Medline

30. Bigler ED, Mortensen S, Neeley ES, et al. Superior temporal gyrus, language function, and autism. Dev Neuropsychol 2007;31:217-38 CrossRef Medline

31. Li W, Qin W, Liu H, et al. Subregions of the human superior frontal gyrus and their connections. Neuroimage 2013;78:46-58 CrossRef Medline

32. Signorelli M, Tiberti A, Valseriati D, et al. Width of the fetal lateral ventricular atrium between 10 and $12 \mathrm{~mm}$ : a simple variation of the norm? Ultrasound Obstet Gynecol 2004;23:14-18 CrossRef Medline

33. Bloom SL, Bloom DD, DellaNebbia C, et al. The developmental outcome of children with antenatal mild isolated ventriculomegaly. Obstet Gynecol 1997;90:93-97 CrossRef Medline

34. Masoller N, Sanz-Cortés M, Crispi F, et al. Mid-gestation brain Doppler and head biometry in fetuses with congenital heart disease predict abnormal brain development at birth. Ultrasound $\mathrm{Ob}$ stet Gynecol 2016;47:65-73 CrossRef Medline

35. Egaña -Ugrinovic G, Sanz-Cortes M, Figueras F, et al. Fetal MRI insular cortical morphometry and its association with neurobehavior in late-onset small-for-gestational-age fetuses. Ultrasound Obstet Gynecol 2014;44:322-29 CrossRef Medline 